\title{
Spin-mixing dynamics in a spin-1 atomic condensate coupled with a molecular condensate
}

\author{
Jing Cheng, ${ }^{1}$ Hui Jing, ${ }^{2}$ and YiJing Yan $^{3}$ \\ ${ }^{1}$ Department of Physics, South China University of Technology, Guangzhou 510640, China \\ ${ }^{2}$ Department of Physics, Henan Normal University, Xinxiang 453007, China \\ ${ }^{3}$ Department of Chemistry, Hong Kong University of Science and Technology, Kowloon, Hong Kong
}

(Received 17 January 2008; published 11 June 2008)

\begin{abstract}
We study the coherent spin-mixing dynamics in a spin-1 atomic condensate coupled with a molecular condensate. A model is proposed to include the photoassociation (PA) interaction in a spinor condensate. If only the $m_{F}=-1$ spin component can be converted into molecules, the combined dynamics of spin-mixing and PA can be described by nonlinearly coupled pendulums. We performed detailed numerical calculations on the antiferromagnetic spin-1 systems. We find the spin-mixing dynamics can be greatly controlled by the PA field. The PA field can enhance or suppress the period and amplitude of the spin oscillations, which provide more degrees of freedom to control the spin-mixing dynamics.
\end{abstract}

DOI: $10.1103 /$ PhysRevA.77.061604

PACS number(s): 03.75.Mn, 05.30.Jp, 03.75.Kk, 34.50.Rk

Soon after the experimental realization of Bose-Einstein condensates (BECs) of cold atoms in a single Zeeman state $[1,2]$, spinor condensates became a very active research topic [3-5]. In a spinor condensate, the atomic internal spin degrees of freedom are accessible. Due to the presence of Zeeman degeneracy and spin-dependent collisions, spinor condensates attract many theoretical studies, such as the groundstate structures [6], domain formation [7], and spin-mixing dynamics [8-10]. Coherent spin oscillations have been experimentally observed in both ferromagnetic and antiferromagnetic spinor condensates [11-13].

Cold molecule physics is an emerging research field [14]. Feshbach resonance (FR) [15] and photoassociation (PA) $[16,17]$ are two main techniques to produce cold molecules from an atomic BEC. Unlike FR, which needs strong magnetic fields, PA is purely optical and has wider applicable ranges. The coherent dynamics of coupled atom-molecule BECs exhibits very rich behaviors, including superchemistry [18], bistability [19], and Rabi oscillations [20], just to name a few. Recently, atom-molecule BEC dark states have been realized experimentally $[21,22]$.

Up to now, all studies on atom-molecule BEC have been concerned with only a single internal atomic state, and there have been no studies on the properties in converting a spinor BEC into a molecular BEC. In this Rapid Communication, we propose a simple model to combine the two active topics: the spinor BEC and the coherent dynamics of coupled atommolecule BECs. We try to determine how the spin-mixing dynamics in a spin-1 BEC can be affected by an optical field through the PA process. Controlling the spin-mixing dynamics is possible in our model.

PA is a kind of optical transition. In a spin-1 BEC, any combinations of spins can be converted into a molecule. To completely describe a PA process of associating two spin-1 atoms into a molecule, a lot of of matter wave fields are needed, three for the atom and more for the molecule. To simplify the problem, we propose a model in which only $m_{F}=-1$ atoms are converted into molecules. As proposed by Ref. [10], applying a small magnetic field can break the degeneracy of the spin-1 atoms. So, the energy of $m_{F}= \pm 1,0$ components can be Zeeman-shifted. If we use a polarized laser to near resonantly couple the transition between the
$F=1$ ground state to the $F=0$ excited state, then the $m_{F}=-1$ component can be efficiently converted into molecules, while the coupling strengths of other PA transitions are very small due to the selection rules and frequency detuning. Figure 1 depicts our model. The atoms are in three different hyperfine spin $F=1$ ground states: $|1,+1\rangle,|1,0\rangle$, $|1,-1\rangle$. Due to the spin-dependent collisions, two atoms in the $|0\rangle$ state can be converted to one $|+1\rangle$ and one $|-1\rangle$ atom, and vice versa. This kind of interaction is responsible for the spin-mixing dynamics. We further introduce another interaction for the PA process to generate a moleculein the $|b\rangle$ state from two $|-1\rangle$ atoms. Under these assumptions and approximations, and using the single-spatial-mode approximation (SMA) [23], we obtain the model Hamiltonian,

$$
\begin{aligned}
H= & E_{+} \hat{a}_{+}^{\dagger} \hat{a}_{+}+E_{0} \hat{a}_{0}^{\dagger} \hat{a}_{0}+E_{-} \hat{a}_{-}^{\dagger} \hat{a}_{-}+E_{m} \hat{b}^{\dagger} \hat{b} \\
& +\left[\Omega(t)\left(\hat{a}_{-}\right)^{2} \hat{b}^{\dagger}+\Omega^{*}(t)\left(\hat{a}_{-}^{\dagger}\right)^{2} \hat{b}\right]+\frac{c_{0}}{2} \sum_{\alpha, \beta} \hat{a}_{\alpha}^{\dagger} \hat{a}_{\beta}^{\dagger} \hat{a}_{\alpha} \hat{a}_{\beta} \\
& +\frac{c_{2}}{2}\left[\left(\hat{a}_{+}^{\dagger}\right)^{2}\left(\hat{a}_{+}\right)^{2}+\left(\hat{a}_{-}^{\dagger}\right)^{2}\left(\hat{a}_{-}\right)^{2}-2 \hat{a}_{+}^{\dagger} \hat{a}_{-}^{\dagger} \hat{a}_{-} \hat{a}_{+}+2 \hat{a}_{+}^{\dagger} \hat{a}_{0}^{\dagger} \hat{a}_{0} \hat{a}_{+}\right. \\
& \left.+2 \hat{a}_{-}^{\dagger} \hat{a}_{0}^{\dagger} \hat{a}_{0} \hat{a}_{-}+2\left(\hat{a}_{0}^{\dagger}\right)^{2} \hat{a}_{+} \hat{a}_{-}+2 \hat{a}_{+}^{\dagger} \hat{a}_{-}^{\dagger}\left(\hat{a}_{0}\right)^{2}\right] .
\end{aligned}
$$

Here, $\Omega(t)=\Omega_{0} \exp \left(-i \omega_{L} t\right)$ is the coupled Rabi frequency of the PA process; $c_{0}$ and $c_{2}$ are interaction coefficients of spin- 1 atoms, which are determined from the $s$-wave scattering length for two spin-1 atoms in different collision chan-

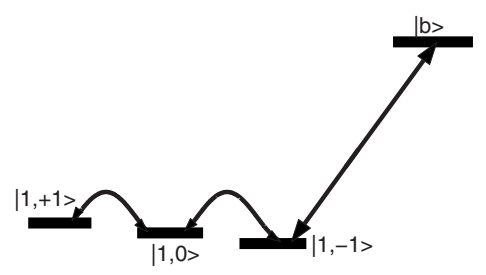

FIG. 1. A model of an $F=1$ spinor BEC coupled with a molecular BEC through PA. $|1,+1\rangle,|1,0\rangle,|1,-1\rangle$ are the atomic spinor states, and $|b\rangle$ is the molecular state. Spin-mixing can occur among the atoms, and $|1,-1\rangle$ atoms can be photoassociated to the molecular state. 
nels [9]. Generally, $c_{0}$ is much larger than $c_{2} . \hat{a}_{s}$ and $\hat{b}$ are the operators that annihilate an atom in the state $|s\rangle$ and a molecule, respectively. $E_{s}=\hbar \omega_{s}$ are the Zeeman-shifted energy of the corresponding atomic or molecular states. Equation (1) conserves the total particles $N=n_{+}+n_{0}+n_{-}+2 n_{m}$. All collisions depending on the molecules are neglected because we have no knowledge of their strengths, and the current studies are restricted in the region with a few molecules.

We use the mean-field method to study the system Eq. (1). With the help of the SMA, we obtain the equations of motion (EOM) of the spinor atomic and molecular matter wave fields,

$$
\begin{aligned}
i \dot{a}_{+}= & {\left[1-2|b|^{2}+c_{2}\left(\left|a_{+}\right|^{2}+\left|a_{0}\right|^{2}-\left|a_{-}\right|^{2}\right)\right] a_{+}+c_{2} a_{0}^{2} a_{-}^{*}, } \\
i \dot{a}_{0}= & {\left[1-\delta-2|b|^{2}+c_{2}\left(\left|a_{+}\right|^{2}+\left|a_{-}\right|^{2}\right)\right] a_{0}+2 c_{2} a_{+} a_{-} a_{0}^{*}, } \\
i \dot{a}_{-}= & {\left[1-2|b|^{2}+c_{2}\left(\left|a_{-}\right|^{2}+\left|a_{0}\right|^{2}-\left|a_{+}\right|^{2}\right)\right] a_{-}+c_{2} a_{0}^{2} a_{+}^{*} } \\
& +\Omega_{0} a_{-}^{*} b,
\end{aligned}
$$

$$
i \dot{b}=\Delta b+\frac{\Omega_{0}}{2} a_{-}^{2},
$$

where $\delta=\left(\omega_{-}+\omega_{+}-2 \omega_{0}\right) / 2$ parametrizes the quadratic Zeeman effect [10], and $\Delta=\left(\omega_{b}-2 \omega_{-}\right)-\omega_{L}$ is the laser detuning between the atom-molecule transition. The rotatingwave approximation is used. In the derivation, we have made several scaling transformations, $a_{+} \sim a_{+} / \sqrt{N} e^{-i \omega_{+} t}, \quad a_{0}$ $\sim a_{0} / \sqrt{N} e^{-i\left(\omega_{0}+\delta\right) t}, \quad a_{-} \sim a_{-} / \sqrt{N} e^{-i \omega_{-} t}, \quad b \sim b / \sqrt{N} e^{-i\left(2 \omega_{-}-\omega_{L}\right) t}$, $\Omega_{0} \sim \Omega_{0} \sqrt{N}, c_{0} \sim c_{0} N, c_{2} \sim c_{2} N$, and the time $t \sim t c_{0} N$. The EOM [Eq. (2)] have two invariants, the total particle number $n_{+}+n_{0}+n_{-}+2 n_{m}=1$, and the generalized magnetization $n_{+}-\left(n_{-}+2 n_{m}\right)=m$.

Similar to Ref. [10], we may use a classical dynamical system to describe our model. Taking $a_{s}=\sqrt{n_{s}} e^{-i \theta_{s}}$, $b=\sqrt{n_{m}} e^{-i \phi}$, and introducing new conjugate variables $x$, $\alpha$ and $y, \beta$, where $x=n_{-}, \alpha=\theta_{+}+\theta_{-}-2 \theta_{0}, \quad y=n_{m}$, and $\beta=\phi-4 \theta_{0}+2 \theta_{+}$, the energy functional of the classical dynamical system is

$$
\begin{aligned}
\mathcal{E}= & \Delta y-\delta(1-2 x-4 y-m)+\frac{1}{2}(1-2 y)^{2}+\frac{c_{2}}{2}(m+2 y)^{2} \\
& +c_{2}(1-m-2 x-4 y)(m+2 x+2 y) \\
& +2 c_{2}(1-m-2 x-4 y) \sqrt{x(m+2 x+2 y)} \cos (\alpha) \\
& +\Omega x \sqrt{y} \cos (\beta-2 \alpha) .
\end{aligned}
$$

This is a model of two coupled nonlinear pendulums. The single pendulum models in Ref. [10] or Ref. [19], for the spin mixing or PA processes only, can be derived as special cases from Eq. (3).

The model Eq. (3) is nonintegrable; analytical solutions do not exist. Therefore, we have to numerically solve Eq. (2) to study how the spin-mixing dynamics are affected by the $\mathrm{PA}$ process. In our numerical calculations, we focus our attention on the antiferromagnetic $\left({ }^{23} \mathrm{Na}\right)$ spin-1 atoms because they have a relative large $c_{2}(\sim 0.03)$ [24]. The magnetic field is selected to ensure $\delta \geq 0.01$ and the linear Zeeman shift
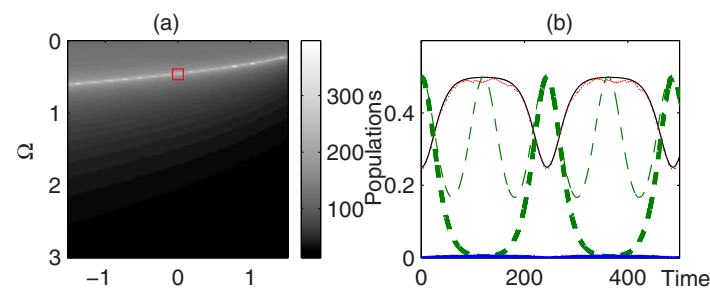

(c)

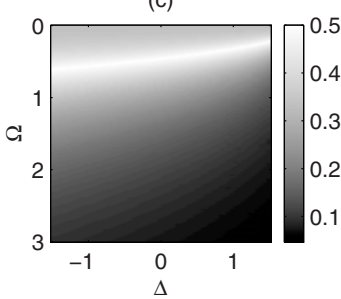

(d)

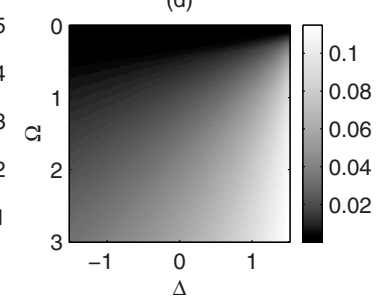

FIG. 2. (Color online) Numerical results for $\delta=0.02$, $f_{0} \equiv\left[a_{+}, a_{0}, a_{-}, b\right]_{t=0}=[1, \sqrt{2}, 1,0] / 2$. (a) The oscillation period of the spin- 0 atoms as a function of $\Omega$ and $\Delta$. (b) The temporal evolution of the populations of the four components with $\Omega=0.46$ and $\Delta=0$. The black thin solid line, $n_{+}(t)$; the green thick dashed line, $n_{0}(t)$; the red thin dotted line, $n_{-}(t)$; the blue thick solid line, $n_{m}(t)$. The green thin dashed line is $n_{0}(t)$ with $\Omega=0$ and $\Delta=0$. (c) The oscillation amplitude of the spin-0 atoms. (d) The oscillation amplitude of the molecules.

$\eta=\left(\omega_{+}-\omega-\right)>50$. The PA parameters are limited with $\Omega_{0}<3$ and $|\Delta|<1.5$. In realistic experiments, the bare $c_{2} N$ can achieve several $\mathrm{Hz}$ to several tens of $\mathrm{Hz}$. In the following, we present results with different parameters $(\delta, \Omega, \Delta)$ and initial values of the four fields $f_{0} \equiv\left[a_{+}, a_{0}, a_{-}, b\right]_{t=0}$. In all calculations, we suppose there is no molecular population initially, i.e., the system is initially prepared as a purely spinor BEC.

In Fig. 2, we study the system initially with atomic population $[0.25,0.5,0.25]$ and they are in phase. The magnetic field is chosen to lead to $\delta=0.02$. Without the PA field, the spinor dynamics of atoms exhibit oscillations [8-10]. Depending on the values of the initial matter wave $\left(f_{0}\right)$, there is a critical magnetic field, corresponding to a critical $\delta_{c}$, at which the oscillation period diverges $[10,13]$. Here, from Fig. 2(a), we can see that there are also some critical PA fields, at which the oscillation period achieves a maximum value. For any given $\Delta$, when $\Omega$ is increased, the oscillation period first increases, reaches a maximum value, and then decreases. In Fig. 2(b), we show an example of the four components when $\Omega=0.46$ and $\Delta=0$. The $m_{F}=1$ and 0 components exhibit large, anharmonic oscillations. The $m_{F}=-1$ and molecular components simultaneously suffer both PA and spin-mixing interactions, and their behaviors are the superposition of the two different interactions. Because the PA interaction $\Omega$ is much larger than the spin-mixing interaction $c_{2}$, the fast oscillation is mainly due to the PA process, and the slowly oscillated envelope is from thespin mixing. Since $m$ is conserved in evolution, the sum of the $m_{F}=-1$ and the molecular components equals the $m_{F}=1$ component in this example. For comparison, we also plot the $m_{F}=0$ evolution when there is no PA field. It has a smaller oscillation period and amplitude. In Figs. 2(c) and 2(d), the oscillation amplitudes of the spin- 0 and molecular components are depicted. 
(a)

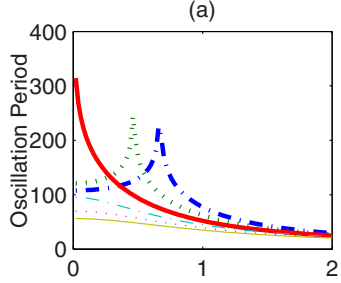

(c)

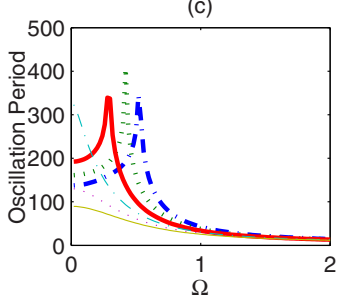

(b)

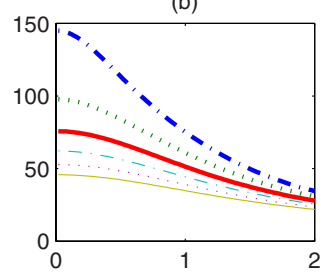

(d)

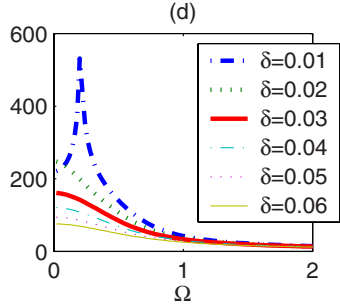

FIG. 3. (Color online) The influence of $\delta$ on the oscillation period, with $\Delta=0$. (a) $f_{0}=[1, \sqrt{2}, 1,0] / 2$. (b) $f_{0}=[1, \sqrt{2},-1,0] / 2$. (c) $f_{0}=[\sqrt{0.05}, \sqrt{0.1}, \sqrt{0.85}, 0]$. (d) $f_{0}=[\sqrt{0.05}, \sqrt{0.1},-\sqrt{0.85}, 0]$.

The spin-0 atoms also exhibit the single peak structure. For large $\Omega$, the PA interaction is more important than the spin mixing, so the $m_{F}=0$ component is likely to be self-trapped. The maximum achievable faction of molecules is a monotonously increasing function of $\Omega$ and $\Delta$. It is easy to understand that increasing $\Omega$ leads to a stronger PA interaction and more molecules converted from the atoms. The collisions between atoms effectively shift the atomic energy, after which a slightly larger positive $\Delta$ will compensate this shift. This causes the oscillation amplitude to become larger, as shown in Fig. 2(d). From this figure, both the oscillation period and the amplitude of the spin-mixing dynamics can be enhanced or suppressed by an appropriate PA field.

Varying the magnetic field (or $\delta$ ), the plots in Fig. 2 will be changed. In Fig. 3(a), we plot the oscillation period $T(\Omega)$ as a function of $\Omega$ for six different values of $\delta$, with $\Delta=0$. When $\delta$ is small, $T(\Omega)$ shows a single-peak structure. For large $\delta, T(\Omega)$ is a decreasing function of $\Omega$. In Fig. 3(a), $\delta_{c}=0.03$. We performed many other numerical simulations to see the influence of $\delta$ on the oscillation period. Several examples are given in Figs. 3(b)-3(d). In Fig. 3(b), $\delta_{c}=0$, all $T(\Omega)$ monotonously decrease with $\Omega$. In Fig. 3(c), $\delta_{c}=0.0394$, we find that the curves with $\delta=0.01,0.02$, and 0.03 have a single-peak structure, and the peak is positioned at small $\Omega$ for large $\delta$. In Fig. 3(d), $\delta_{c}=0.0146$, only $\delta$ $=0.01$ is single-peaked. All these simulations suggest the following conjecture: if $\delta<\delta_{c}, T(\Omega)$ has a single-peak structure, with the peak approaching $\Omega=0$ as $\delta$ increases; if $\delta>\delta_{c}, T(\Omega)$ is a decreasing function of $\Omega$. We cannot prove this conjecture, but numerical evidence strongly supports it.

Until now, we have neglected the loss of molecules in all calculations. In current PA experiments, the directly generated molecules are unstable. Some schemes, such as Raman PA and STIRAP [25-28], have been suggested to convert the unstable molecules into stable ones. In fact, because the twomode PA model used in Eq. (1) can be derived from a more realistic three-level Raman PA model, as shown in Ref. [25], the molecular state in our simulations can be considered as a stable molecular state. In Fig. 4, we phenomenologically include a decay term $-\gamma b$ on the right side of the molecular
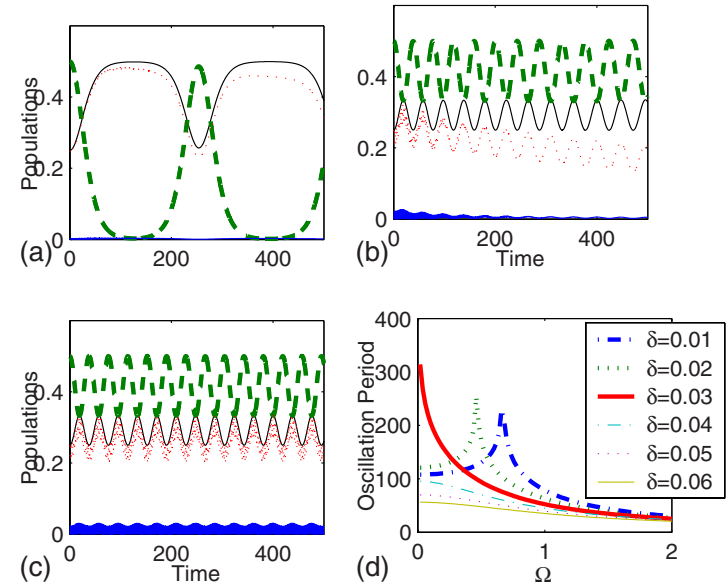

FIG. 4. (Color online) Simulated results when there is molecule decay, $\gamma=0.01$. (a) The same as Fig. 2(b), $\gamma=0.01$. The black thin solid line, $n_{+}(t)$; the green thick dashed line, $n_{0}(t)$; the red thin dotted line, $n_{-}(t)$; the blue thick solid line, $n_{m}(t)$. (b) The temporal evolution of the populations of the four components with $\Omega=1.5$ and $\Delta=0, \gamma=0.01$. (c) The same as (b), but $\gamma=0$. (d) Oscillation periods for different $\delta$ when there is decay.

EOM in Eq. (2). Setting $\gamma=0.01$, comparing Fig. 4(a) with Fig. 4(b), we see that the oscillation is slightly damped. To see the effects of molecule decay more clearly, we choose a larger $\Omega=1.5$, and compare Fig. 4(b) with Fig. 4(c). When including the decay term, the fast oscillation of the $m_{F}=-1$ and molecular components are greatly suppressed, and their amplitudes are damped. On the other hand, the evolution of the $m_{F}=0$ or 1 component is almost unchanged. Figure $4(\mathrm{~d})$ is the oscillation period of the $m_{F}=0$ component for six different values of $\delta$. Compared with Fig. 3(a), their behaviors are very similar, with only small variations at large $\Omega$. Thus, even though there is molecular loss, the effects of PA on spin mixing should still be observable, at least for not too long an evolution time.

Finally, we give some realistic estimates on our results. The parameters used in this paper follow from Ref. [13]. Explicitly, the density of the atoms in the BEC is about $8.6 \times 10^{13} \mathrm{~cm}^{-3}, c_{2}$ is $127 \mathrm{~Hz}$ in our calculations, and a unit time corresponds to $0.24 \mathrm{~ms}$. A unit amplitude of $\Omega$ or $\Delta$ or $\delta$ will be $4200 \mathrm{~Hz}$. All these values are not far from the realistic experimental situations. There are some technical difficulties in realizing our model, such as the need for highly frequency stabilized laser sources and fine frequency detuning, which may present great challenges to the experimentalists.

In conclusion, we have proposed a model to study the spin-mixing dynamics in a spin-1 atomic condensate coupled with a molecular condensate through PA. Using a small magnetic field to break the atomic Zeeman degeneracy, we study only the $m_{F}=-1$ spin component to be converted into molecules. The combined dynamics of spin-mixing and PA can be described by nonlinearly coupled pendulums. Focusing on the antiferromagnetic spin-1 BEC, we performed detailed studies on how the spin-mixing dynamics in a spin-1 BEC can be affected by the PA field. Depending on the amplitude 
of the magnetic field, the PA field can enhance or suppress the period and amplitude of the spin oscillations. Our work provides a kind of optical method to control the spin mixing dynamics.
J.C. is supported by the National Natural Science Foundation of China (Grants No. 10404031 and No. 10774047) and the SCUT BaiRen Program. Y.J.Y. is supported by the Research Grants Council of the Hong Kong Government.
[1] M. H. Anderson, J. R. Ensher, M. R. Matthews, C. E. Wieman, and E. A. Cornell, Science 269, 198 (1995).

[2] K. B. Davis, M.-O. Mewes, M. R. Andrews, N. J. van Druten, D. S. Durfee, D. M. Kurn, and W. Ketterle, Phys. Rev. Lett. 75, 3969 (1995).

[3] J. Stenger, S. Inouye, D. M. Stamper-Kurn, H.-J. Miesner, A. P. Chikkatur, and W. Ketterle, Nature (London) 396, 345 (1998).

[4] T.-L. Ho, Phys. Rev. Lett. 81, 742 (1998).

[5] T. Ohmi and K. Machida, J. Phys. Soc. Jpn. 67, 1822 (1998).

[6] W. Zhang, S. Yi, and L. You, New J. Phys. 5, 77 (2003).

[7] Q. Gu and H. Qiu, Phys. Rev. Lett. 98, 200401 (2007).

[8] C. K. Law, H. Pu, and N. P. Bigelow, Phys. Rev. Lett. 81, 5257 (1998).

[9] H. Pu, C. K. Law, S. Raghavan, J. H. Eberly, and N. P. Bigelow, Phys. Rev. A 60, 1463 (1999).

[10] W. Zhang, D. L. Zhou, M. S. Chang, M. S. Chapman, and L. You, Phys. Rev. A 72, 013602 (2005).

[11] M.-S. Chang et al., Nat. Phys. 1, 111 (2005).

[12] J. Kronjager, C. Becker, P. Navez, K. Bongs, and K. Sengstock, Phys. Rev. Lett. 97, 110404 (2006).

[13] A. T. Black, E. Gomez, L. D. Turner, S. Jung, and P. D. Lett, Phys. Rev. Lett. 99, 070403 (2007).

[14] Eur. Phys. J. D 31 (2) (2004), special issue of Ultracold Polar Molecules: Formation and Collisions, edited by J. Doyle, B. Friedrich, R. V. Krems, and F. Masnou-Seeuws.

[15] E. A. Donley, N. R. Claussen, S. T. Thompson, and C. E. Wieman, Nature (London) 417, 529 (2002).
[16] R. Wynar et al., Science 287, 1016 (2000).

[17] K. M Jones, E. Tiesinga, P. D Lett, and P. S. Julienne, Rev. Mod. Phys., 78, 483 (2006).

[18] D. J. Heinzen, R. Wynar, P. D. Drummond, and K. V. Kheruntsyan, Phys. Rev. Lett. 84, 5029 (2000).

[19] Y. Wu and R. Cote, Phys. Rev. A 65, 053603 (2002).

[20] A. Ishkhanyan, G. P. Chernikov, and H. Nakamura, Phys. Rev. A 70, 053611 (2004).

[21] K. Winkler, G. Thalhammer, M. Theis, H. Ritsch, R. Grimm, and J. H. Denschlag, Phys. Rev. Lett. 95, 063202 (2005).

[22] R. Dumke, J. D. Weinstein, M. Johanning, K. M. Jones, and P. D. Lett, Phys. Rev. A 72, 041801(R) (2005).

[23] In our work, since the atomic number is not consvered, the mode function is determined from the usual Schrödinger equation for a single atom, rather than the generally used nonlinear Schrödinger equation. This method was used in O. E. Mustecaphoglu, Wenxian Zhang, and L. You, Phys. Rev. A 75, 023605 (2007)

[24] W. X. Zhang, Ph.D. desseration, Georgia Institute of Technology, 2005 (unpublished).

[25] M. Kostrun, M. Mackie, R. Cote, and J. Javanainen, Phys. Rev. A 62, 063616 (2000).

[26] M. Mackie, R. Kowalski, and J. Javanainen, Phys. Rev. Lett. 84, 3803 (2000).

[27] P. D. Drummond, K. V. Kheruntsyan, D. J. Heinzen, and R. H. Wynar, Phys. Rev. A 65, 063619 (2002).

[28] H. Y. Ling, H. Pu, and B. Seaman, Phys. Rev. Lett. 93, 250403 (2004). 